\title{
Derin evrişimli sinir ağı modellerinin açık kaynak kodlu yazılım platformlarında tasarımının değerlendirilmesi
}

\author{
H. Selcuk Nogay ${ }^{\mathrm{a}, *}$, Tahir Cetin Akıncı ${ }^{\mathrm{b} 1}$, Serhat Şeker ${ }^{\mathrm{b} 2}$ \\ a Kayseri Üniversitesi, Mustafa Çıkrıkçıŏlu MYO, Elektrik ve Enerji Bölümü, Kayseri, Türkiye. \\ $\mathrm{b}$ İstanbul Teknik Üniversitesi, Elektrik Mühendisliği Bölümü, İstanbul, Türkiye.
}

İstanbul Sabahattin Zaim Üniversitesi Fen Bilimleri Enstitüsü Dergisi (2020) 2 (3): $94-98$

https://doi.org/10.47769/izufbed.859937

(iD) $O R C I D{ }_{1}^{1} 0000-0001-9105-508 X ;{ }^{2}$ 0000-0002-4657-6617; ${ }^{3} 0000-0001-5816-2211$

\begin{tabular}{l} 
YAYIN BİLGİSİ \\
\hline Yayın geçmişi: \\
Gönderilen tarih: 13 Ocak 2021 \\
Kabul tarihi0: 12 Nisan 2021 \\
\end{tabular}

Anahtar kelimeler:

Açık kaynak kodlu (AKK)

Kapalı kaynak kodlu (KKK)

Yazılım

DESA

Öğrenme aktarımı

\begin{tabular}{l}
\hline ÖZET \\
\hline Derin evrişimli sinir ağları, iki boyutlu verilerin kullanıldığı, en popüler ve en yaygın derin \\
öğrenme yöntemlerinden birisidir. Özellikle lisans ve lisansüstü öğrencilerin derin öğrenme \\
yöntemlerini özgürce uygulayabilecekleri ve geliştirebilecekleri yeni derin öğrenme modelleri \\
tasarlayabilecekleri, bu konudaki deneyimlerini arttırabilecekleri ortamlara maliyetsiz ve kolayca \\
ulaşabilmeleri, bu gençlerin insanlığa ve bilime hizmet edebilecek bilgi, beceri ve deneyime sahip \\
olmaları açısından çok önemlidir. Açık kaynak kodlu yazılım platformları eğer üniversitelerde \\
ders olarak okutulursa ve öğrencilerin öğrencilik dönemleri boyunca eğitilebilecekleri bir ortama \\
kavuşmaları açısından son derece büyük avantaja sahiptir. Ne var ki günümüzde üniversiteler \\
MATLAB gibi ticari yazılımların lisansını aldıklarında araştırmacıların ulaşabildiği ancak \\
öğrencilerin ulaşamadığı derin öğrenme uygulama ortamları ortaya çıkmaktadır. MATLAB derin \\
öğrenme uygulamalarının gerçekleştirilmesi açısından maliyetli olması dışnda önemli bir \\
dezavantajı olmayan bir kapalı kaynak kodlu ticari bir yazılımdır. Bu çalışmada derin evrişimsel \\
sinir ağı modellerinin açık kaynak kodlu yazılım platformlarında tasarımı kaynak araştırması \\
yapılarak ele alınmış ve MATLAB ile kıyaslanmıştır. Açık kaynak kodlu yazılım platformları ile \\
DESA uygulamalarının kolay ulaşılabilir olmasısı sağlamak ve gençler arasında popülaritesinin \\
arttırılabilmesi için üniversitelerin müfredat programlarına ders olarak konulması gerekliliği \\
sonucuna varılmıştır.
\end{tabular}

\section{Evaluation of the design of deep convolution neural network models using open source software platforms}

\begin{tabular}{l}
\hline ARTICLE INFO \\
\hline Article history: \\
Received: 13 January 2021 \\
Accepted: 12 April 2021
\end{tabular}

\section{Key words:}

Open source coded (OSC)

Closed source coded (CSC)

software

DCNN

Transfer learning

\section{Giriş}

Literatürde, kaynak kodu herkes tarafindan özgürce

\begin{abstract}
Deep convolutional neural networks (DESA) is one of the most popular and common deep learning method using two-dimensional data. It is especially important for undergraduate and graduate students to have free and easy access to environments where they can freely apply and develop deep learning methods, design new deep learning models, and have knowledge, skills and experience that can serve humanity and science. Open source software platforms have a great advantage if they are taught as a course in universities and in terms of providing an environment where students can be educated during their student period. However, nowadays, when universities obtain the license of commercial software such as MATLAB, deep learning application environments that researchers can reach but cannot reach by students emerge. MATLAB is a commercial software with closed source code that does not have any significant disadvantages other than being costly in terms of realizing deep learning applications. In this study, the design of deep convolutional neural network models on open source software platforms has been handled and compared with MATLAB. It was concluded that open source software should be included in the curriculum of universities in order to make DCNN applications easily accessible and to increase their popularity among young people with open source software platforms.
\end{abstract}

kullanılabilir ve geliştirilebilir olan yazılımlar açık kaynak kodlu (AKK) yazılımlar olarak adlandırılmaktadır (Walt 2003). AKK yazılımlar kullanıcıya yazılımı değiştirme ve 
kendi gereksinimlerine uygun bir şekilde uyarlama özgürlüğ̈̈ sağlamaktadır. Bilgisayar teknolojisinin ve ona bağlı olarak yazılım teknolojisinin ilerlemesi ile bilim dünyasının uğraştı̆̆ ve halen uğraşmakta olduğu birçok problemin çözümü için yazılımlar ve algoritmalar geliştirilmektedir. $\mathrm{Bu}$ gelişme sürecinin son dönemlerinde yapay sinir ağları ile başlayan ve birçok yapay zeka yöntemini de içine alarak makine öğrenmesi adı ile günümüze kadar gelen bütün algoritma ve yöntemler AKK yazılım platformlarının geliştirilerek artmasıyla günümüzün derin öğrenme ortaya çıkmasına ve gelişmesine vesile olmuştur.

Derin öğrenme modellerinin iki boyutlu (2-D) görüntü verilerinin kullanıldığ 1 en fazla tercih edileni ve en popüler olanı derin evrişimli sinir ağları (DESA) modelleridir. Son zamanlarda DESA birçok problemin çözümünde ve birçok bilimsel çalışmada yaygın olarak uygulanmaktadır. Bu uygulamaların bazıları MATLAB gibi ticari olan kapalı kaynak kodlu (KKK) yazılım ortamlarında, bazıları ise Pyhton, Keras ve Tensorflow gibi açık kaynak kodlu yazılım ortamlarında gerçekleştirilmektedir. Bu çalışmada açık kaynak kodlu yazılım ortamları ile bir DESA modeli tasarlayabilmenin ve geliştirerek uygulayabilmenin olasılığı, pozitif ve negatif yönleri ile ele alınmıştır. $\mathrm{Bu}$ amaçla çalışmada hem MATLAB ortamında hem de açık kaynaklı yazılım ortamlarında gerçekleştirilmiş DESA modelleri incelenmiştir.

\section{2. İlgili çalışmalar}

Son teknolojide gerek AKK ve gerekse KKK yazılım ortamlarında gerçekleştirilmiş birçok bilimsel çalışma gerçekleştirilmiştir. AKK yazılım ortamlarında yapılan DESA uygulamalarına örnek gösterilebilecek bir çalışmada, Ahmed ve diğerleri, insan yüzü tanıma amacıyla, Tensorflow kütüphanesinden yararlanılarak önceden eğitilmiş (pretrained) klasik dört adet DESA modelini öğrenme aktarımı yaklaşımından faydalanarak kıyasladılar (Ahmed vd., 2020). Bir AKK yazılım platformlarından olan Pyhton ortamında gerçekleştirilen başka bir uygulamada, bir robota yerleştirilmiş bir kamera ile çekilen resimleri ya da başka bir ifadeyle bir robotun bir ortamı keşfederken çekmiş olduğu resimleri bölümlere ayırmak için, DESA modeli kullanılmıştır (Rodrigues vd., 2017). Başka bir AKK çalışmasında bir DESA modeli Keras ortamında el yazısı tanıma amaciyla gerçekleştirilmiştir (Arora ve Bhatia 2018). AKK yazılım platformlarından bir diğeri olan Tensorflow altyapısı kullanarak yapılan bir başka DESA uygulaması, açık kaynak kodlu yazılımların geliştirilmesine yardımeı olabilmek, kaynak kodlarının sınıflandırılması ve yürütme zamanının en aza indirgenmesi maksadıyla gerçekleştirilmiştir (Barchi vd., 2021). Günümüzün en büyük salgını olan koronavirüs (Covid19) salgını için, derin öğrenme ve tıbbi görüntü işleme çalışmaları için gerçekleştirilmiş, AKK yazılım ortamlarında tasarlanan DESA uygulamaları giderek sayısını arttırmaktadır (Bhattacharya vd., 2020).

DESA modelleri de diğer bütün yapay zeka modelleri gibi ancak veri seti ile uygulanabilir olduğu için, açık kaynak teknolojisinin veri biliminin gelişiminde kullanımı (Boehmke et al 2020), sadece AKK yazılım ortamlarında değil MATLAB gibi ticari KKK yazılım ortamlarında gerçekleştirilen DESA modelleri için de büyük bir gerekliliktir. Birçok araştırmacı veya öğrenci için halka açık veri tabanlarının AKK yazılım ortamları veya kütüphaneleri sayesinde geliştirilmesi ve ulaşılabilir olması hem AKK yazılımlara dair farkındalığın artmasına yardımcı olabilir, hem de daha gelişmiş ve büyük veri setleri ile eğitilen ileri DESA uygulamalarının artmasına ve yaygınlaşmasına katkı sağlayabilir.

AKK yazılım ortamlarının, kullanıcının ustalığına ve tecrübesine de bağlı olarak, uzaktan görüntü algılama ve görüntü işlemede (Cresson 2019), moleküler modellemede (Cummings ve Gilmer 2019), DESA simülatörü tasarımında (Dogaru ve Dogaru 2019), nesne tanımada (Duth ve Raj 2018), elektrik makinalarında arıza tespitinde (Jiao vd., 2020), elektrik güç sistemlerinde arıza sınıflandırmada (Rai vd., 2020), yazılım mühendisliğinde kaynak kodun geliştirilmesi ve yeniden kullanılabilir hale getirilmesinde (Jindal ve Mittal 2020), model tabanlı mimarilerin gelişiminde ve metamodel (modelin modeli) sinıflandırmada (Nguyen vd., 2021), grafiksel bir boyutlu (1-D) veriler ile teknik uygulamalarda (Kiranyaz vd., 2021), yüz tanımada (Qiao and Ma 2018; Yuan vd., 2017), biyolojik görüntü sınıflandırmada (Qin vd., 2020), insansız hava araçlarında görüntü tanımada (Quiñonez vd., 2020), çoklu odaklama ile görüntü birleştirmede (Multifocus Image Fusion) (Yang vd., 2019), konuşma tanımada (Lin vd., 2018), tıpta, diyabetik retinopati siniflandirmasinda (Gayathri vd., 2020) ve seviyelendirmesinde (Ghosh vd., 2017), pömoni teşhisinde (Jakhar ve Hooda 2018) ve daha bir çok alanda büyük faydalar sağlayabildiği görülmektedir. Yukardaki çalışmaların hepsi de AKK derin öğrenme kütüphanelerinden yararlanılarak gerçekleştirilmiş çalışmalardır. DESA modellerinin literatürde en fazla yararlandığı AKK platformları veya kütüphaneleri; Theano, Torch, Tensorflow ve Keras dır.

AKK yazılım ortamlarının sunduğu en önemli imkanlardan birisi, daha önce tasarlanmış ve eğitilmiş (pre-trained) olan klasikleşmiş DESA modellerini, öğrenme aktarımı (transfer learning) yaklaşımı sayesinde bazı katmanlarında değişiklikler yaparak tekrar yeni bir amaç için kullanabilme imkanıdır. Ne var ki AKK yazılım ortamlarında bir DESA modeli tasarlamak ve uygulamak için kullandığınız kütüphanenin yazılım dilini bilmeniz ve hatta kendinizi o yazılım dilin de çok geliştirmeniz gerekmektedir. Ancak günümüzün en yaygın olarak kullanılan KKK yazılım örneği olan MATLAB bazı yönleri ile AKK yazılımların sunduğu firsatlardan daha fazla firsat sunabilmekte ve bir DESA mimarisi açısından yeni tasarımlar geliştirmeyi daha kolay hale getirmektedir. Öte yandan MATLAB vb ticari KKK yazılım platformlarının maliyeti ve güncellenme ihtiyacı, özellikle üniversite öğrencilerinin derin öğrenme eğitimi için cazip olmaktan çıkmaktadır. Birçok araştırmacı çalıştıkları üniversite bünyesinde MATLAB “deep learning toolbox" ile AKK yazılım ortamlarına benzer şekilde, klasik önceden eğitilmiş DESA modellerini öğrenme aktarımı yaklaşımı sayesinde uygulayabilmekte ve bağımsız bir şekilde kendi DESA tasarımlarını geliştirerek birçok problemin çözümüne bilimsel yaklaşım sunmaktadırlar. Günümüzde AKK yazılım platformlarının sunduğu değiştirme, geliştirme ve uyarlama imkanlarının aynısına MATLAB "deep learning toolbox" ortamında sahip olarak, tıp alanında farklı kanser tiplerinin sınıflandırılmasında (Nogay 2018), akciğerdeki skuamöz hücreli karsinomun yerini tahmin etmek için (Nogay ve Akıncı 2018), akciğer kanseri seviyesinin tespitinde 
(Nogay 2017) ve tiplerinin sinıflandırmasında (Nogay vd., 2018a), baş-boyun kanserinin seviyesinin tespitinde (Nogay vd., 2018b) ve otizm teşhisi amacıyla birçok çalışmada uygulanmaktadır (Nogay ve Adeli, 2020). Mühendislik ve teknik alanlarda da AKK yazılım platformlarının imkanlarına benzer şekilde, öğrenme aktarımı yaklaşımı sayesinde, elektrik ark kaynak makinelerinin optimum çalışma bölgelerinin tespitinde DESA mimarisi kullanılmıştır (Nogay ve Akınc1 2020).

\section{AKK ve KKK yazılım platformlarında DESA tasarımı}

Derin öğrenme tasarımlarının yapılabildiği, en fazla kullanılan AKK yazılım platformları, Pyhton alt yapısında Theano, Torch, Tensorflow ve Keras kütüphaneleridir. Her birey bu plotformlara ulaşabilir veya kendi bilgisayarlarına kurabilir. AKK yazılım platformlarının en önemli avantajı herkesin herhangi bir maliyet olmaksızın bu yazılım ortamlarına ulaşabilmesidir. Ancak bu platformlardan sadece o yazılım dilinin eğitimini almış kişiler yararlanabilmektedir. DESA tasarımı yapabilmek için sadece makine öğrenmesi alt yapısı ile ilgili temel ve teorik bilgilere sahip olmak yetmez aynı zamanda eğer AKK ortamında DESA tasarımı yapacaksanız ilave olarak kullanacağınız AKK yazılım platformunu öğrenmeniz ve hatta kullanacağınız dilin eğitimini almanız gerekmektedir. AKK web uygulamaları büyük veri setlerine ulaşmayı kolaylaştırarak DESA tasarımlarını daha varyasyonal ve geniş bir ortamda gerçekleştirmeyi sağlayabilir. Jupyter Notebook, canlı kod, metin ve görselleştirmeleri içerecek şekilde belgeler oluşturmaya ve paylaşmaya izin veren bir AKK web uygulaması olarak örnek gösterilebilir. Şekil 1' de Jupyter Notebook ortamında Pyhton dili ile Tensorflow kütüphanesinde yapılmakta olan bir DESA tasarımı sürecinin bir parçası gösterilmektedir.

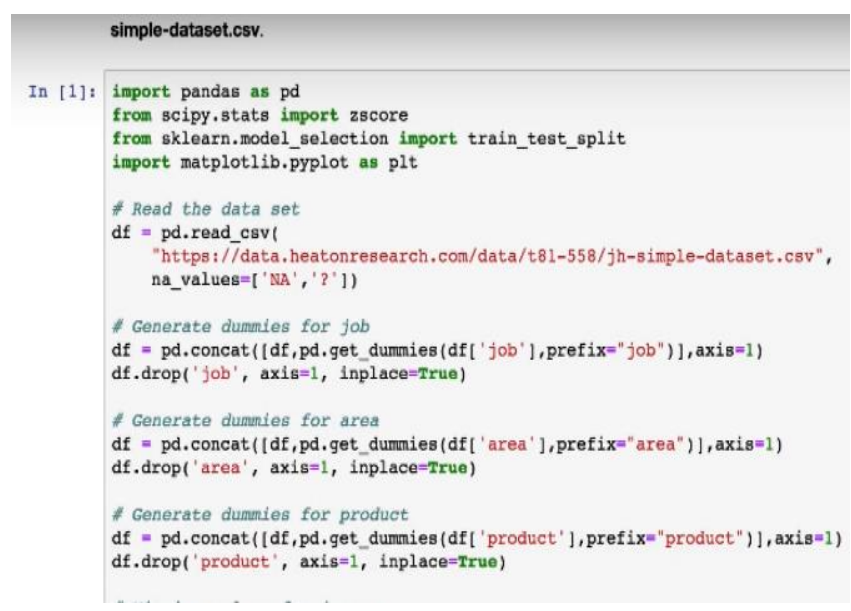

Şekil 1. Jupyter Notebook penceresinde Pyhton dili ile Tensorflow kullanarak DESA tasarımından bir kesit

KKK yazılım ortamlarında ise özgürce bir DESA tasarım imkanı, sağlayıcı firmaya göre değişmektedir. MATLAB özellikle yapay zeka uygulamaları için çok geniş bir çerçeve sunarken, derin öğrenme uygulaması ve tasarımı için neredeyse AKK yazılım platformlarındaki kadar bir geliştirme ve tasarlama özgürlüğünü “deep learning toolbox" sayesinde sunmaktadır. Önceden eğitilmiş (pre-trained) modelleri tekrar kullanabilmek için öğrenme aktarımı (transfer learning) yaklaşımının kolaylıkla uygulanabilmesi, sıfırdan bir DESA tasarımı için görselliğin önplana çıktığı materyallerin kullanılması önemli avantajlar sağlamaktadır. Ancak bu avantajlar MATLAB' in açık kaynak özelliğinden ileri gelmektedir. Şekild 2 de Matlab ortamındaki "deep learning toolbox" ve içerdiği önceden eğitilmiş klasik DESA modelleri gösterilmektedir.

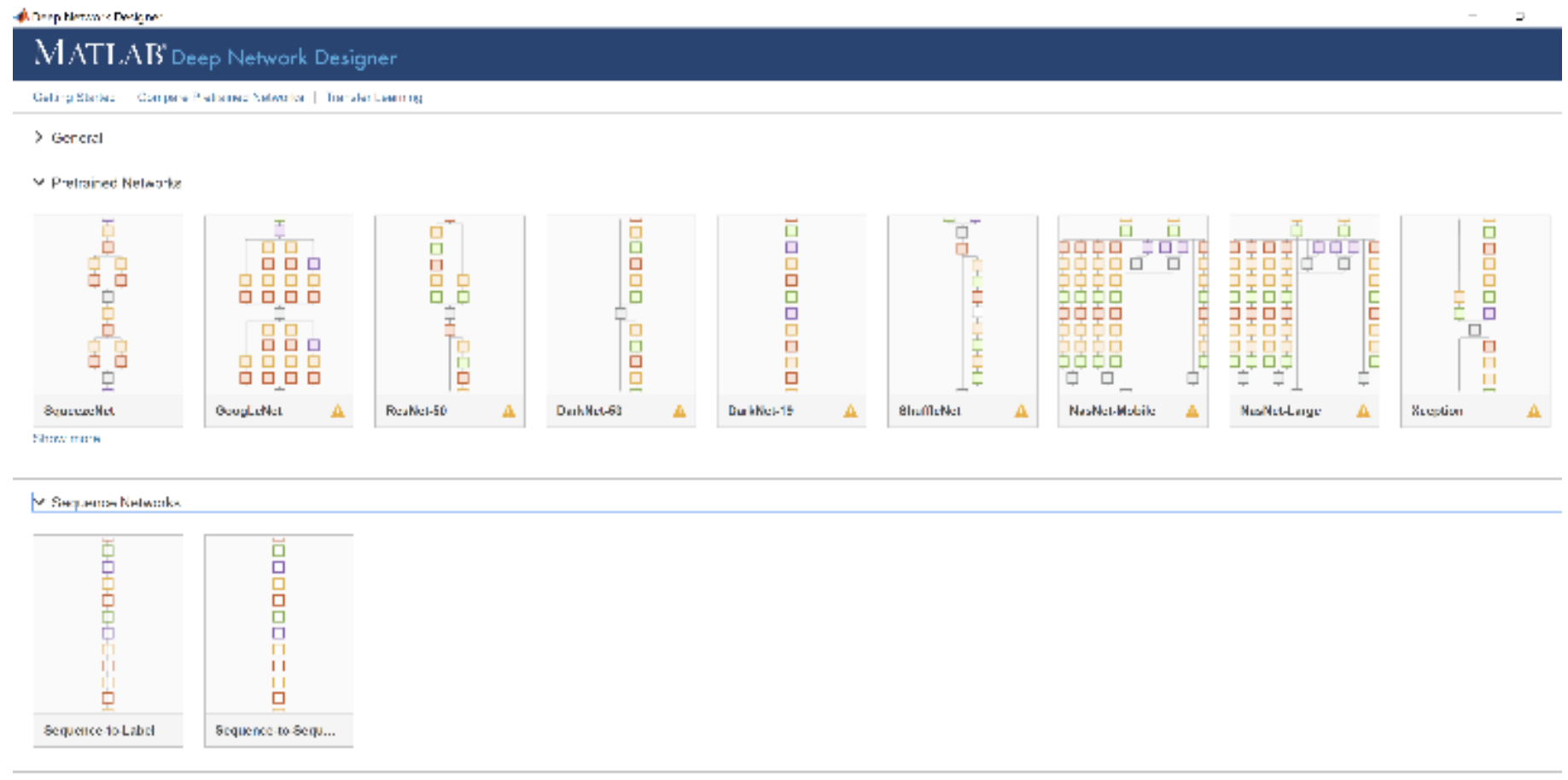

Şekil 2. MATLAB ortamında DESA tasarımı için Deep Network Designer penceresi 
MATLAB ortamında "Deep Network Designer" penceresi ile bir DESA tasarımı sadece programın kendi yardım dökümanlarından yararlanılarak kolaylıkla yapılabilir. AKK yazılım ortamlarında olduğu gibi ayrıca bir eğitim almak veya özellikle bir yazılım diline tam hakim olmak gerekmez. Ancak makine öğrenmesi yöntemleri için temel teorik bilgiye sahip olmak gerekir. DESA tasarımında, KKK yazılım olarak MATLAB "Deep Learning Toolbox" yada yeni adiyla "Deep Network Designer" baz alınarak, AKK olarak da en çok kullanılan Theano, Torch, Tensorflow ve Keras kütüphaneleri baz alınarak Tablo 1 oluşturulmuştur. Tablo 1 de DESA tasarımında AKK ve KKK yazılımlarının avantaj ve dezavantajları gösterilmektedir.

Tablo1. DESA tasarımında AKK ve KKK yazılımların avantaj ve dezavantajları

\begin{tabular}{|c|c|c|}
\hline & $\begin{array}{l}\text { AKK (Theano, Torch, } \\
\text { Tensorflow Keras) }\end{array}$ & KKK (MATLAB) \\
\hline 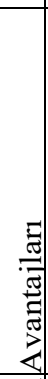 & 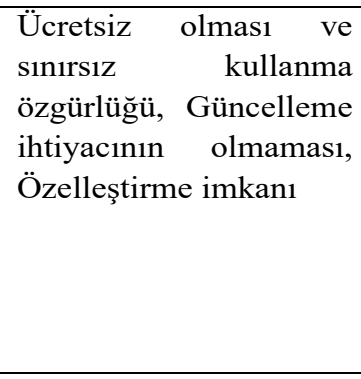 & $\begin{array}{l}\text { Sağlayıcının tüm yazılım } \\
\text { bileşenlerinin sorunsuz } \\
\text { çalışma garantisi vermesi, } \\
\text { geniş tasarım ve geliştirme } \\
\text { imkanı tanıması, görsel } \\
\text { olarak uzun ve karmaşık } \\
\text { kodlamalara } \\
\text { kalmadan kolay tasarım } \\
\text { imkanı tanıması }\end{array}$ \\
\hline 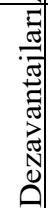 & $\begin{array}{l}\text { Kurulum ve kullanım } \\
\text { için tecrübe ve eğitim } \\
\text { gereksinimi, kullanım } \\
\text { zorluğu, aşırı derecede } \\
\text { eklentilere dayalı olması }\end{array}$ & $\begin{array}{l}\text { Maliyetinin yüksek olması, } \\
\text { Güncelleştirme } \\
\text { gereksinimi ve yer } \\
\text { kaplaması }\end{array}$ \\
\hline
\end{tabular}

\section{Sonuç ve değerlendirme}

AAK yazılım platformları ile gerçekleştirilmiş ve literatürde yerini almış olan birçok başarılı DESA uygulaması vardır. AAK yazılım platformlarında gerçekleştirilen ve birçok problemin çözümüne katkı sağlayan bu DESA uygulamalarının bir kısmı önceden eğitilmiş (pre-trained) DESA modellerinin AAK yazılım ortamının verdiği avantaj ile öğrenme aktarımı tekniğinden yararlanılarak, bazı katmanlarının değiştirilerek adapdasyonu sayesinde ve diğer bir kısmı da sıfırdan tasarlanan DESA modelleri sayesinde yapılmıştır. Ancak KKK yazılım platformlarından, DESA tasarım ve uygulamalarında bariz bir şekilde en yaygın olarak kullanılan "MATLAB - Deep learning toolbox" esas alındığında çok sayıda DESA uygulamasının literatürde yer aldığ 1 bir gerçektir.

AAK yazılım platformlarının kullanıcının bilgisayara kurulumunu yapması ve uygulamaya başlaması deneyimsiz ve yazılım dili eğitimi olmayan bireyler için zorlu bir süreç olabilir. KKK platformu olarak sadece MATLAB düşünüldüğünde bu süreç daha kolaydır. DESA uygulaması KKK platformları içerisinde en yaygın olarak sadece MATLAB ortamında uygulanabildiği için MATLAB esas alınarak değerlendirme yapmaktayı. Ancak şu da bir gerçektir ki AAK yazılım ortamları üniversitelerde lisans veya lisans üstü seviyesinde ders olarak okutulabilir ve DESA uygulamaları öğrencilere lisans üstü derslerde yaptırılabilir. $\mathrm{Bu}$ durumda hem kurulum deneyimi olan ve hem de yazılım diline hakim bir öğrenci kitlesi için AAK yazılım platformları bedava ve özgür bir tasarım ortamı sunacaktır.

\section{Kaynaklar}

Ahmed, T., Das, P., Ali, F., Mahmud, F. (2020). A Comparative Study on Convolutional Neural Network Based Face Recognition. 2020 11th International Conference on Computing, Communication and Networking Technologies (ICCCNT), IEEE, 1-3 July, Kharagpur, India.

Arora, S., Bhatia, M.P.S. (2018). Handwriting recognition using Deep Learning in Keras. International Conference on Advances in Computing, Communication Control and Networking (ICACCCN2018), IEEE, 12-13 October, Greater Noida (UP), India.

Barchi, F., Parisi, E., Urgese, G., Ficarra, E., Acquaviva, A. (2021). Exploration of Convolutional Neural Network models for source code classification. Engineering Applications of Artificial Intelligence, 97, 104075.

Bhattacharya, S., Maddikunta., P. K. R., Pham, Q.V., Thippa Reddy Gadekallu., T. R., Krishnan, S. R., Chowdhary, C. L., Alazab, M., Piran, J. (2020). Deep learning and medical image processing for coronavirus (COVID-19) pandemic: A survey. Sustainable Cities and Society, https://doi.org/10.1016/j.scs.2020.102589, xxx, (xx).

Boehmke, B., Hazen, B., Boone, C. A., Robinson, J. L. (2020). A data science and open source software approach to analytics for strategic sourcing, International Journal of Information Management. 54, 102167.

Cresson, R. (2019). A Framework for Remote Sensing Images Processing Using Deep Learning Techniques. IEEE Geoscience and Remote Sensing Letters, 16, (1):25-29.

Cummings, P. T., Gilmer, J. B. (2019). Open-source molecular modeling software in chemical engineering. Current Opinion in Chemical Engineering, 23:99-105.

Dogaru, R., Dogaru, I. (2019). BCONV-ELM: Binary Weights Convolutional Neural Network Simulator based on Keras/Tensorflow, for Low Complexity Implementations. 6th International Symposium on Electrical and Electronics Engineering (ISEEE), 18-20 Oct. Galati, Romania.

Duth, S., Raj, S. (2018). Object Recognition in Images using Convolutional Neural Network. Proceedings of the Second International Conference on Inventive Systems and Control (ICISC), 19-20 Jan., Coimbatore, India.

Gayathri, S., Varun, P. G., Palanisamy, P. (2020). A lightweight CNN for Diabetic Retinopathy classification from fundus images. Biomedical Signal Processing and Control, 62, 102115.

Ghosh, R., Ghosh, K., Maitra, S. (2017). Automatic Detection and Classification of Diabetic Retinopathy stages using CNN. 4th International Conference on Signal Processing and Integrated Networks (SPIN), 2-3 February, Noida, India.

Jakhar, K., Hooda, N. (2018). Big Data Deep Learning Framework using Keras: A Case Study of Pneumonia Prediction. 4th International Conference on Computing Communication and Automation (ICCCA) 14-15 Dec. 2018. Greater Noida, India.

Jiao, J., Zhao, M., Lin, J., Liang, K. (2020). A comprehensive review on convolutional neural network in machine fault diagnosis. Neurocomputing, (417): 36-63. 
Jindal, R., Mittal, S.K. (2020). Software reusability metrics estimation for improving stability by clustering base convolution neural network. Materials Today: Proceedings, https://doi.org/10.1016/j.matpr.2020.09.615, xxx, (xx).

Kiranyaz, S., Avci, O., Abdeljaber, O., Ince, T., Gabbouj, M., Inman, D.J. (2021). 1D convolutional neural networks and applications: A survey. Mechanical Systems and Signal Processing, 151, 107398.

Lin, B.Y., Huang, H.S., Sheu, R. K., Chang, Y.S. (2018). Speech recognition for people with dysphasia using convolutional neural network. IEEE International Conference on Systems, Man, and Cybernetics (SMC), 7-10 Oct. Miyazaki, Japan.

Nguyen, P.T., Ruscio, D., Pierantonio, A., Rocco, J.D., Iovino, L. (2021). Convolutional neural networks for enhanced classification mechanisms of metamodels. The Journal of Systems \& Software, 172, 110860.

Nogay, H. S. (2018). Classification Of Different Cancer Types By Deep Convolutional Neural Networks. Balcan Journal of Electrical\&Computer Engineering, 5: 56-59.

Nogay, H. S., Akıncı, T. C. (2018). A Convolutional Neural Network Application For Predicting The Locating Of Squamous Cell Carcinoma In The Lung. Balkan Journal of Electrical \& Computer Engineering, 6: 207-210.

Nogay, H. S. (2017). Deep Convolutional Neural Networks To Detect Lung Cancer Stage", The Journal of Cognitive Systems, 2: 33-36.

Nogay, H. S., Akınc1, T. Ç., Erdemir, G. (2018a). A Convolutional Neural Network Application For The Classification Of Lung Cancer Types. Academic Journal Industrial Technologies, 5:7-12,

Nogay, H. S., Akıncı, T. Ç., Erdemir, G. (2018b) "Estimation Of Head \& Neck Cancer Stage By Using Deep Convolutional Neural Networks. Academic Journal Industrial Technologies, 5: 13-19.

Nogay, H. S., Adeli, H. (2020). Machine learning (ML) for the diagnosis of autism spectrum disorder (ASD) using brain imaging, Reviews In The Neurosciences, DOI: $10.1515 /$ revneuro-2020-0043, 1-17.

Nogay, H. S., Akıncı T. Ç. (2020). Classification of operation cases in electric arc welding wachine by using deep convolutional neural networks, Neural Computing \& Applications, https://doi.org/10.1007/s00521-020-05436-y, 1-14.

Rai, P., Londhe, N.D., Raj, R. (2020). Fault classification in power system distribution network integrated with distributed generators using CNN. Electric Power Systems Research, https://doi.org/10.1016/j.epsr.2020.106914, xxx(xx):xx.

Rodrigues, C. A. S. P., Vinhal, C., Cruz, G. (2017). Fully convolutional networks for segmenting images from an embedded camera. 2017 IEEE Latin American Conference on Computational Intelligence (LA-CCI), IEEE, 8-10 November, Arequipa, Peru.

Qiao, S., Ma, J. (2018). A Face Recognition System Based on Convolution Neural Network. Chinese Automation Congress (CAC), IEEE, 30 Nov.-2 Dec. Xi'an, China.

Qin, J., Pan, W., Xiang, X., Tan, Y., Hou, G. (2020). A biological image classification method based on improved CNN. Ecological Informatics, 58, 101093.

Quiñonez, Y., Carmen Lizarraga, C., Peraza, J., NZatarain, O. (2020). Image recognition in UAV videos using convolutional neural networks. The Institution of Engineering and Technology IET Software, 14 (2):176-181.

Walt, S. (2003). Free/Open Source Software Development Practices in the Computer Game Community. University of CaliforniaInstitute for Software Research, http://www.ics.uci.edu/ redmiles/ics221-FQ03/paper.
Yang, Y., Nie Z., Huang, S., Lin, P., Wu, J. (2019). Multilevel Features Convolutional Neural Network for Multifocus Image Fusion. IEEE Transactions on Computational Imaging, 5(2): 262-273.

Yuan, L., Qu, Z., Zhao, Y., Zhang, H., Nian, Q. (2017). A Convolutional Neural Network based on TensorFlow for Face Recognition. 2017 IEEE 2nd Advanced Information Technology, Electronic and Automation Control Conference (IAEAC), 25-26 March, Chongqing, China. 